\title{
PERFORMANCE OF INERTIAL MASS CONTROLLERS FOR ULTRA-LIGHTWEIGHT FOOTBRIDGES: A CASE STUDY
}

\author{
Carlos M. C. Renedo ${ }^{1}$, Iván M. Díaz ${ }^{1}$, Justin M. Russell ${ }^{2}$ and Stana Živanović ${ }^{3}$ \\ ${ }^{1}$ Universidad Politécnica de Madrid, Department of Continuum Mechanics and Theory of Structure, \\ ETSI Caminos, Canales y Puertos. \\ Madrid, Spain \\ e-mail: carlos.martindelaconcha@upm.es, ivan.munoz@upm.es \\ ${ }^{2}$ University of Warwick, School of Engineering \\ Coventry, United Kingdom \\ e-mail: J.Russell.3@warwick.ac.uk \\ ${ }^{3}$ University of Exeter, College of Engineering, Mathematics and Physical Sciences \\ Exeter, United Kingdom \\ e-mail: S.Zivanovic@exeter.ac.uk
}

Keywords: Structural control, inertial vibration controllers, lightweight structures, humanstructure interaction.

\begin{abstract}
The increasing use of lightweight materials in construction, such as fiber reinforced polymer (FRP) composites or aluminum, together with rising architectural tendencies towards slender and stunning layouts are enabling to dramatically decrease the weight of contemporary structures. This fact involves a positive reduction of the structural carbon footprint, but it poses a challenge to complying with vibration serviceability limit state (VSLS) under human-induced loading. Indeed, ultra-lightweight structures (with relatively low inherent mass and damping) may undergo vertical vibration in a broader frequency band than those built in the past and be prone to human-structure interaction (HSI), which should be considered when assessing VSLS of these structures.

Instead of adding structural mass or performing expensive structural stiffening to rectify the vibration problem, the integration of smart damping strategies could be an optimum solution that has additional benefits of preserving lightweight nature of these structures. This paper presents a case study of vibration control of an ultra-lightweight FRP truss footbridge. This work investigates the influence of three types of inertial mass controllers (passive, semi-active and active) on the vibration control of the bridge in the presence, as well as in the absence, of HSI. It was found that active vibration control was the less deteriorated when considering HSI in comparison with passive and semi-active control strategies.
\end{abstract}




\section{INTRODUCTION}

The contemporary structural design framework is evolving towards a state of greater creative freedom. Nowadays, designers can make use of a wide variety of tools in order to satisfy the increasingly demanding requirements for new structures. The use of lightweight materials such as fiber reinforced polymers (FRP) or aluminium, have enabled development of "minimum weight" structures. Sizing of structures types with moderate strength demands and strict vibration serviceability requirements (e.g. footbridges and composite floor systems) is now governed by vibration criteria rather than ultimate limit state [1]. However, substantial reduction of structural self-weigh, is to be achieved, requires integration of vibration control measures in the design stage in order to satisfy VSLS under Human-induced loading.

Up to now, human-induced vibrations of lightweight structures were mainly caused by resonant behaviours of certain vibration modes located within the high-energy frequency content of dynamic loading generated by humans. For example, traditionally, lightweight steel footbridges with vertical natural frequencies up to $5 \mathrm{~Hz}$ were considered prone to excessive vibration in resonance excitation by one of the first two harmonics of dynamic force generated by humans. In the case of lightweight steel floor systems, this resonant vibration has been usually related to the third harmonic of the human loading [2].

Ultra-lightweight structures may additionally undergo undesired vibrations due to either non-resonant responses excited by lower harmonics of the human loading, or resonant loading related to higher and less energetic harmonics of the human loading. Hence, for FRP or aluminium structures even higher modes up to or above $10 \mathrm{~Hz}$ could respond considerably [3]. Furthermore, whilst the mass of a human in classic steel lightweight structures is much lower than the structural modal mass, this is no longer the case in ultra-lightweight structures. Indeed, human body mass cannot be simply modelled as equivalent dead load. Instead, their interaction with the structural vibration have to be accounted for. Thus, to properly asses the VSLS in ultra-lightweight structures, it is necessary to model humans as interactive dynamic systems. A simple way to achieve this is to model the human as a Mass-Spring-Damper-Actuator system connected to the structure [4].

Future structures will need to be sustainable in terms of material usage and maintenance, and serviceable in terms of static deflection and dynamic comfort. Fortunately, there are many strategies to minimize the dynamic response of a structural system. Among these strategies, inertial controllers are the most widespread damping technology applied to "lively" civil engineering structures [5]. They consist of an inertial mass that applies counteract forces on the structure. They have an advantage of not needing a reference support to react so they can be simply placed on the structural point to be controlled. In practice, inertial controllers can be implemented in three different ways according to the nature of the control: passive, semiactive or active. The passive devices, most commonly known as Tuned Vibration Absorbers (TVAs), are able to damp resonant vibrations when tuned to a single problematic natural frequency of the structure [6]. The Semi-active Tuned Vibration Absorbers (STVAs) are able to adjust the stiffness or damping properties of the controller in real time, by means of smart dampers (usually magneto-rheological dampers). They can control several closed vibration modes as well as vibration modes with time-varying modal parameters [7]. Active Vibration Absorvers (AVAs) make use of actuators for introducing counteract control forces to the lively structure in real time, allowing to mitigate both resonant and non-resonant responses, even over a relatively broad frequency range [8]. 
These three control systems can be studied and designed as a feedback system, so the design problem is set to be a closed-loop system. Up to now, many design strategies based on feedback control have been proposed, however, from the authors knowledge, none of them have yet considered the influence of the HSI as another element which also feeds back the structure to be controlled. When considering HSI, the dynamic properties of the structure, change, an so, the inertial controllers which were designed without taking into account this effect could be degraded. This paper intends to asses the influence of the HSI on the performance of three inertial controllers applied to an existing FRP laboratory footbridge, designed to be as lively as possible under human-induced dynamic loading [3].

The remainder of the paper is organized as follows. First the structure will be described in Section 2. Section 3 outlines the closed-loop system model that incorporates HSI. Section 4 provides selected results on the performance of the three inertial mass controllers in presence and absence of HSI. Finally, Section 5 provides conclusions.

\section{STRUCTURE}

The main aims of designing the studied FRP bridge was to produce a laboratory facility exceptionally responsive to dynamic excitation by human walking and enable research in pedestrian-structure dynamic interaction (Figure 1). To achieve this aim, it was set that the bridge should withstand vibration amplitude of up to $100 \mathrm{~mm}$, on top of the static deflection (of $47.5 \mathrm{~mm}$ for the adopted design solution) due to self-weight.

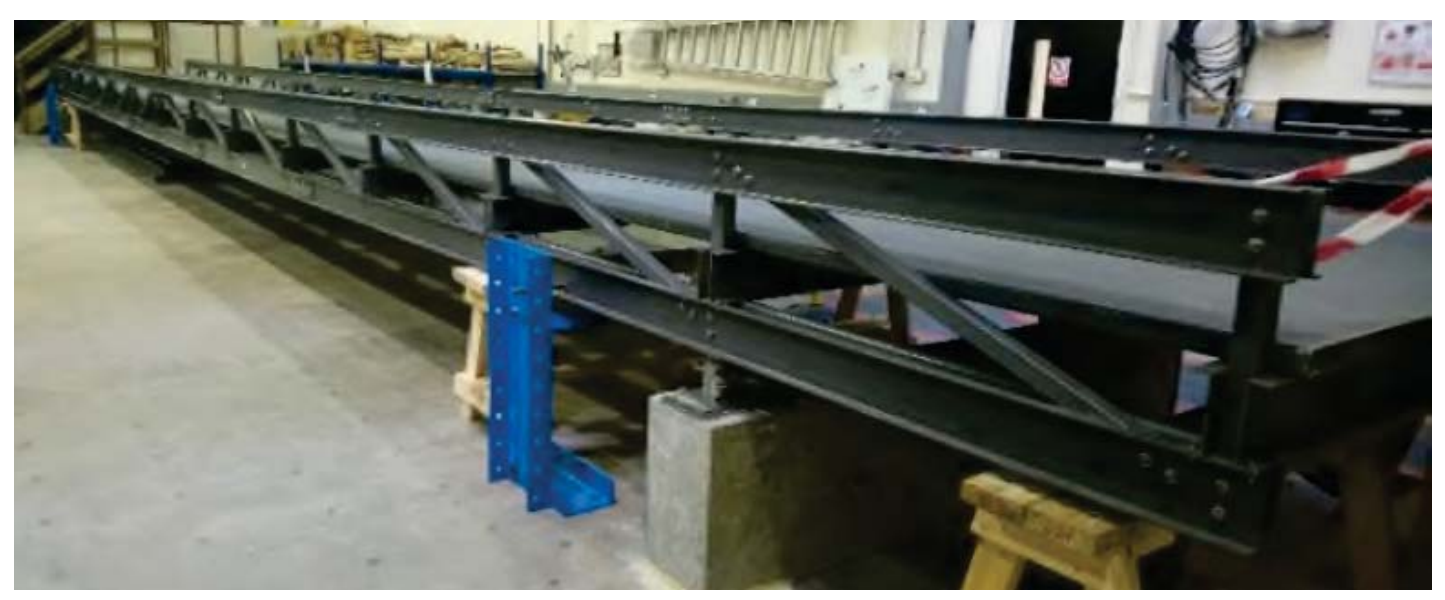

Figure 1: FRP Laboratory Footbridge.

The truss structure with $16.8 \mathrm{~m}$ span and $1.4 \mathrm{~m}$ overhangs at both ends, is made of three types of FRP structural elements as depicted in Figure 2. The bottom chords are simply supported using four steel bearings, including a roller at one end. All connections are executed using stainless steel bolts. Total mass of the bridge is $1400 \mathrm{~kg}$ only. More detailed description of the bridge is available in the paper by Russell et al. 2019 [3]. 
Elevation view

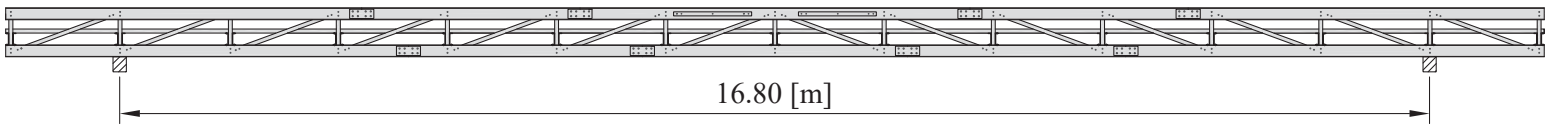

Plan view

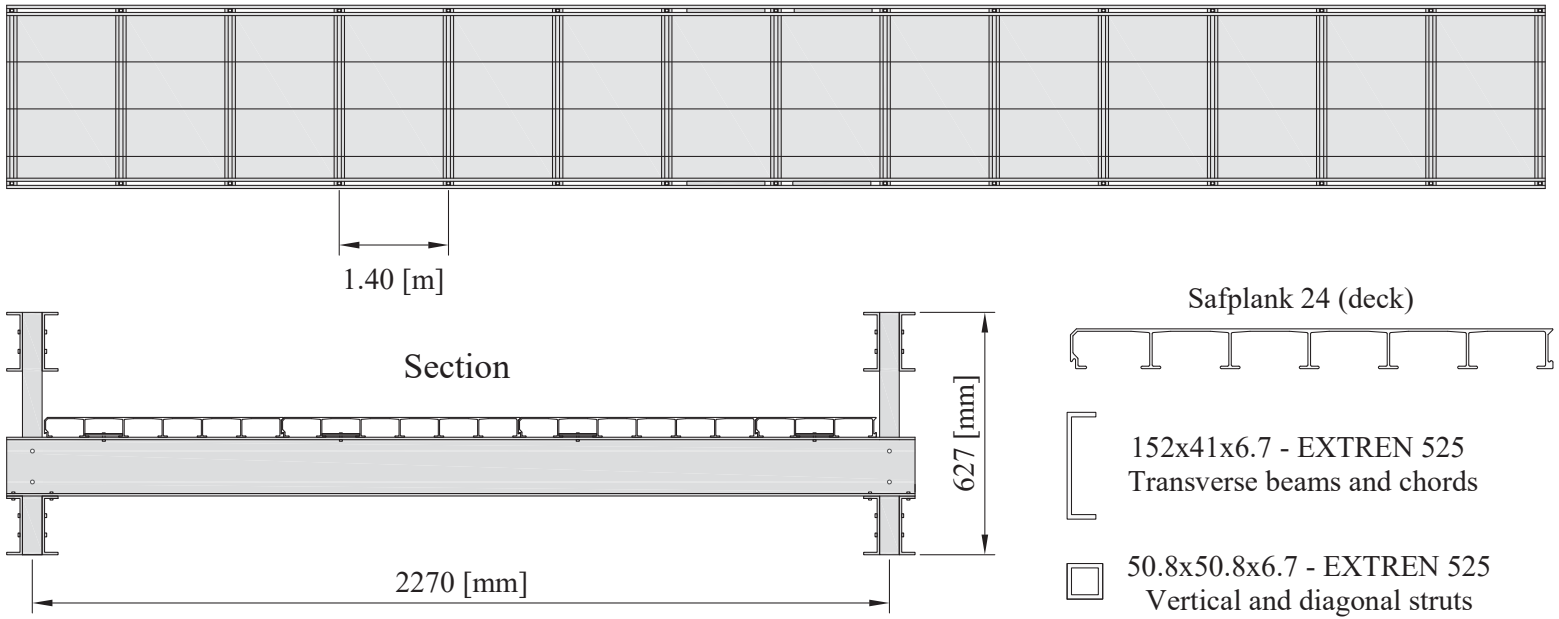

Figure 2: GFRP Laboratory Footbridge: geometrical layout.

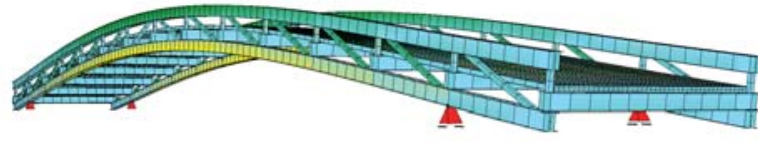

(a) Flexural Mode at $2.5 \mathrm{~Hz}$

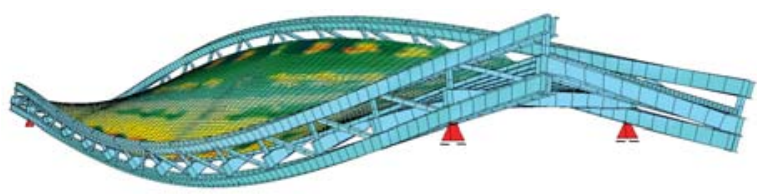

(b) Torsion Mode at $3.4 \mathrm{~Hz}$

Figure 3: First two vibration modes of the structure.

The first two vibration modes have natural frequencies at $2.5 \mathrm{~Hz}$ (first vertical flexural mode) and at $3.4 \mathrm{~Hz}$ (first torsion mode), as shown in Figure 3. It is expected this bridge can achieve vertical acceleration up to around $10 \mathrm{~m} / \mathrm{s}^{2}$ under a single pedestrian walking to excite resonance in the first vibration mode at $2.5 \mathrm{~Hz}$. Indeed, the maximum permitted dynamic deflection of $100 \mathrm{~mm}(\operatorname{span} / 170)$ at $2.5 \mathrm{~Hz}$ would correspond to a peak acceleration of almost $25 \mathrm{~m} / \mathrm{s}^{2}$. In practice, these vibrations are likely to be much lower due to vibration attenuation effects caused by walker-footbridge interaction. Therefore, the primary aim of designing a bridge that can resist the stress under single walker exciting the resonance and, at the same time, be lively was executed successfully [10].

Note that the bridge has not been designed to fulfil the usual design requirement of satisfying the Ultimate Limit State under a live load of $5 \mathrm{kN} / \mathrm{m}^{2}$, as loading of about $500 \mathrm{~kg} / \mathrm{m}^{2}$ by people is not representative for this (and many other) footbridges. The bridge can accommodate several people working on the deck to set up the tests. The deflection due to a single $75 \mathrm{~kg}$ person standing at mid-span is $6.5 \mathrm{~mm}$, and therefore deflection due to a few people walking on the bridge at the same time is much less than the $100 \mathrm{~mm}$ limit. 
This laboratory facility employs much less material than a structure that would comply with VSLS. For this study, an acceleration of $1 \mathrm{~m} / \mathrm{s}^{2}$ has been considered the target value for the control systems. To computationally test the implemented control measures, a single person bouncing loading scenario is utilised.

\section{INERTIAL MASS CONTROLLERS AND HUMAN-STRUCTURE INTERACTION}

This paper focus on the control of the most responsive first flexural vibration mode at $2.5 \mathrm{~Hz}$. This simplification means that this is only a first step in our study known that ultra-lightweight structures tend to display multi-mode dynamic responses excited by several resonant and nonresonant harmonics. The model for general control scheme considered can be divided in three parts: the structure, the human and the inertial controller.

The human and the inertial controller can be modelled as two different elements feeding back to the main structure and changing the system's dynamic properties (and consequently the dynamic response). In this section, the three parts of the dynamic model are described in detail.

\subsection{The structural model}

The case study footbridge has been modelled as an equivalent mass-spring-damper system, which represents the vertical dynamic response of the first flexural mode at mid span, with a mass of $650 \mathrm{~kg}$, a natural frequency of $2.5 \mathrm{~Hz}$, and a damping ratio of $0.94 \%$ [10]. The transfer function between the acceleration response and the excitation force is as follows:

$$
G_{s}(s)=\frac{s^{2} X(s)}{F_{h}(s)}=\frac{1 / m_{s} s^{2}}{s^{2}+2 \omega_{s} \zeta_{s} s+\omega_{s}^{2}}
$$

where $s=j \omega$ is the Laplace variable, $\omega_{s}=2 f \pi f_{s}$ is the circular natural frequency of the structure $(\mathrm{rad} / \mathrm{s}), f_{s}$ is the responding natural frequency in $\mathrm{Hz}$ and $m_{s}$ and $\zeta_{s}$ are the mass $(\mathrm{kg})$ and damping ratio of the first vibration mode at mid-span. Moreover, $F_{h}(s)$ is the Laplace transform of the external human force acting on the structure and $s^{2} X_{s}(s)$ is the Laplace transform of the structural acceleration, Where $X_{s}(s)$ denotes the Laplace transform of the structural displacement. Figure 4 shows the structure block diagram.

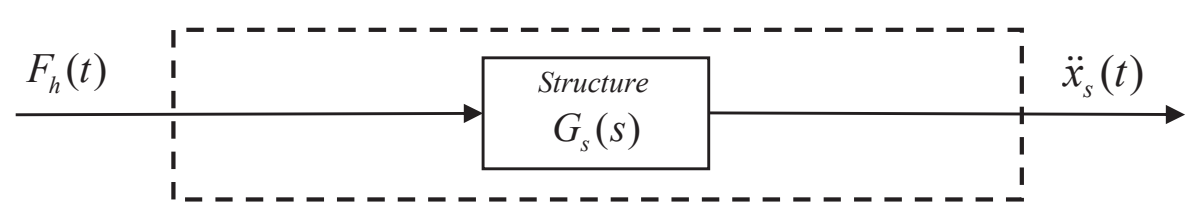

Figure 4: Block diagram of the structure without HSI.

\subsection{The human model}

First, a non-interactive human model is considered. Given the high accelerations expected (as shown in Section 4), it is reasonable to think that the interaction between the human and the 
structure will have significant influence on structural vibration. To account for this, the second model is an interactive model that includes the dynamics of the human.

For the non-interactive model, a dynamic bouncing force measured using a laboratory force plate is used to represent the human on the structure as depicted in Figure 4.

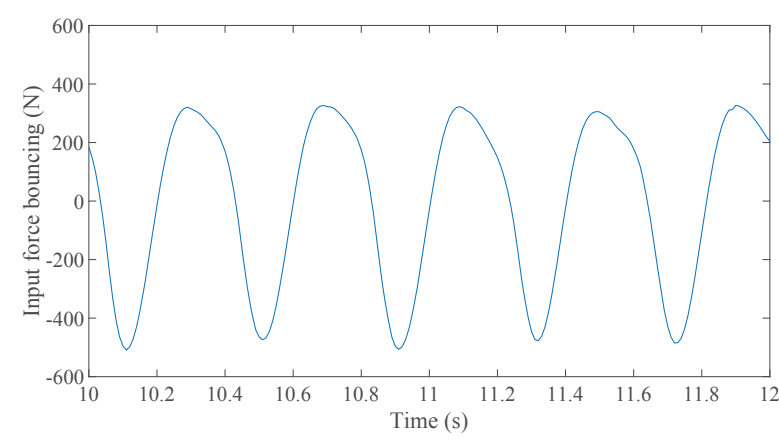

(a) Non-interactive bouncing force at $2.5 \mathrm{~Hz}$.

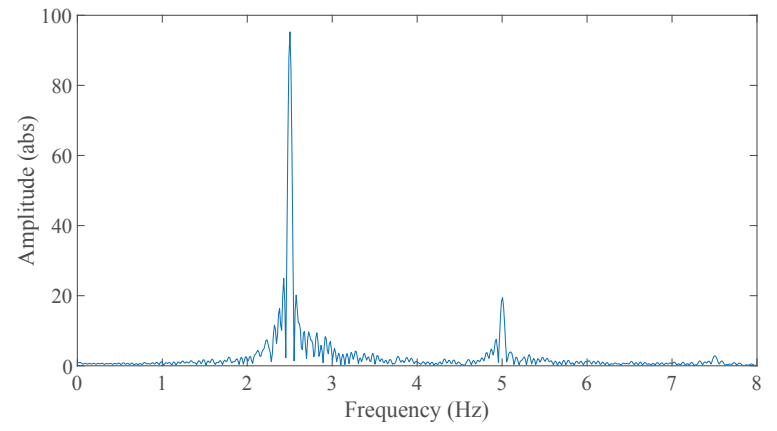

(b) Non-interactive bouncing force spectrum.

Figure 5: Dynamic component of non-interactive bouncing force $F_{h}$ at $2.5 \mathrm{~Hz}$ measured in a laboratory force plate.

Two force waveforms have been applied in the non-interactive simulations: one at resonance conditions of $2.5 \mathrm{~Hz}$ (Figure 5) and the other, out of resonance at $2 \mathrm{~Hz}$. Each force record is 20 s long.

The interactive model considers the dynamics of the human body using a Mass-SpringDamper-Actuator system attached to the structure [11]. Thus, the human is defined by means of its body's natural frequency $f_{h}$, damping ratio $\zeta_{h}$ and mass $m_{h}$. In addition, a harmonic force generated by the human muscles is also accounted for via a pair of action-reaction forces acting simultaneously on both the footbridge and the human, here named as human interactive force or $F_{h i}$.

When considering HSI, the dynamic analysis of the new system (structure + human) is performed assuming that this interaction can be modelled as a closed-loop in which the human feeds back to the main structure. This feedback loop is easily deducted from the force balance illustrated in Figure 6, which is governed by the following equations:

$$
\begin{gathered}
F_{t}=k_{h}\left(x_{h}-x_{s}\right)+c_{h}\left(\dot{x_{h}}-\dot{x_{s}}\right) \\
-m_{h} \ddot{x_{h}}=\left(F_{t}-F_{h i}\right) \\
m_{s} \ddot{x_{s}}+c_{s} \dot{x_{s}}+k_{s} x_{s}=\left(F_{t}-F_{h i}\right)
\end{gathered}
$$

where $x_{h}$ is the human displacement, $k_{h}=\omega_{h}^{2} m_{h}$ is the stifness of the human model, $w_{h}=$ $2 \pi f_{h}$ is the natural circular frequency of the human and $c_{h}=2 \omega_{h} m_{h} \zeta_{h}$ is the viscous damping of the human. Additionally, $x_{s}$ is the structural displacement, $k_{s}=\omega_{s}^{2} m_{s}$ is the structure's stiffness and $c_{s}=2 \omega_{s} m_{s} \zeta_{s}$ is the viscous damping of the structure. $F_{t}$ is the transmitted passive force between the structure and the human. 

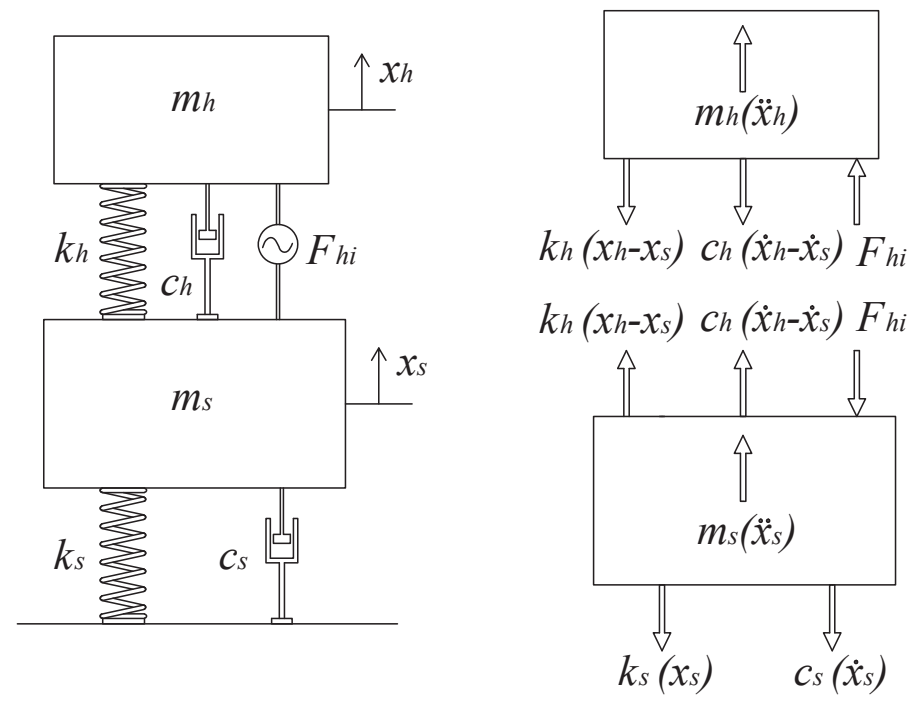

Figure 6: Interactive human model and force equilibrium for each degree of freedom.

The human parameters considered are: mass $m_{h}$ of $66 \mathrm{~kg}$, natural frequency $f_{h}$ of $2.3 \mathrm{~Hz}$ and a damping ratio $\zeta_{h}$ of $25 \%$. Additionally, the muscle force generated by the human modeled as an actuator force $F_{h i}$ has a forcing frequency of either $2.5 \mathrm{~Hz}$ or $2.0 \mathrm{~Hz}$ while its amplitude is $25 \%$ of the human weight:

$$
F_{h i}(t)=Q \alpha \sin (2 \pi f t)
$$

where $Q$ is the human weight taken of $660 \mathrm{~N}$ and $\alpha=0.25$.

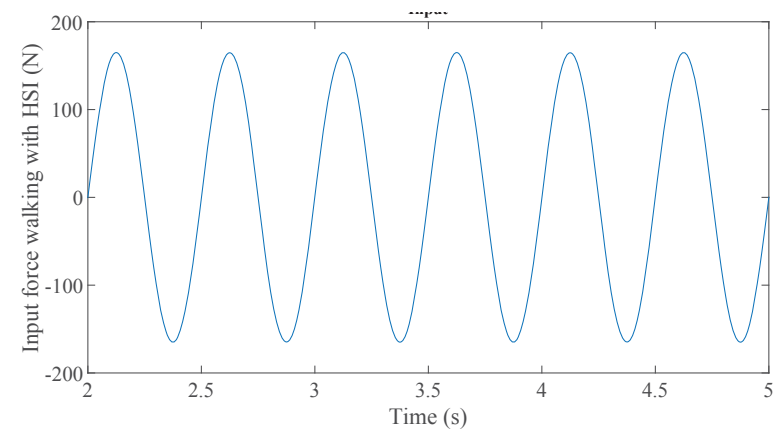

(a) Dynamic interactive bouncing force at $2.5 \mathrm{~Hz}$.

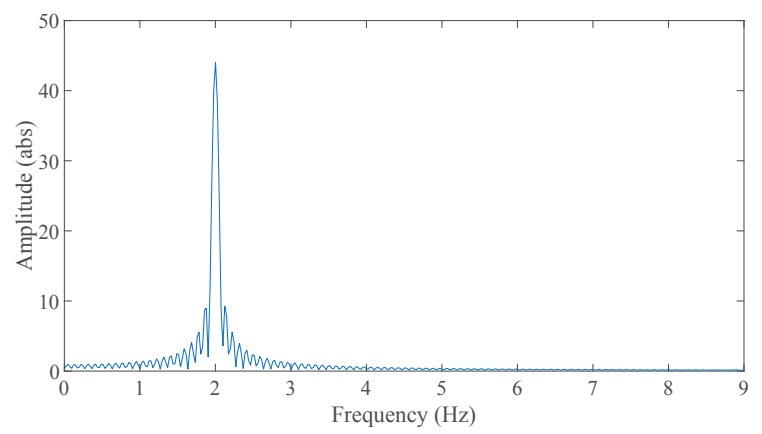

(b) Dynamic interactive bouncing force spectrum.

Figure 7: Human-actuator bouncing force model $F_{h i}$ at $2.5 \mathrm{~Hz}$.

Figure 7 shows the force exerted by the human actuator for bouncing at resonance when accounting for HSI. Note that the amplitude of $F_{h i}$ is lower than the amplitude of $F_{h}$ used for the non-interactive loading model (Figure 5). Finally, the block diagram for the described system is shown in Figure 8. This diagram makes use of the structure's transfer function described in Equation 1 apart from two additional transfer functions used for modelling the interaction: 


$$
\begin{gathered}
G_{h}(s)=\frac{s^{2} X_{h i}(s)}{F_{h i}(s)}=\frac{s^{2}}{m_{h} s^{2}+c_{h} s+k_{h}}=\frac{1 / m_{h} s^{2}}{s^{2}+2 \omega_{h} \zeta_{h} s+\omega_{h}^{2}} \\
G_{H S I}(s)=\frac{s^{2} X_{h-H S I}(s)}{s^{2} X_{s}(s)}=\frac{c_{h} s+k_{h}}{m_{h} s^{2}+c_{h} s+k_{h}}=\frac{2 \omega_{h} \zeta_{h} s+\omega_{h}^{2}}{s^{2}+2 \omega_{h} \zeta_{h} s+\omega_{h}^{2}}
\end{gathered}
$$

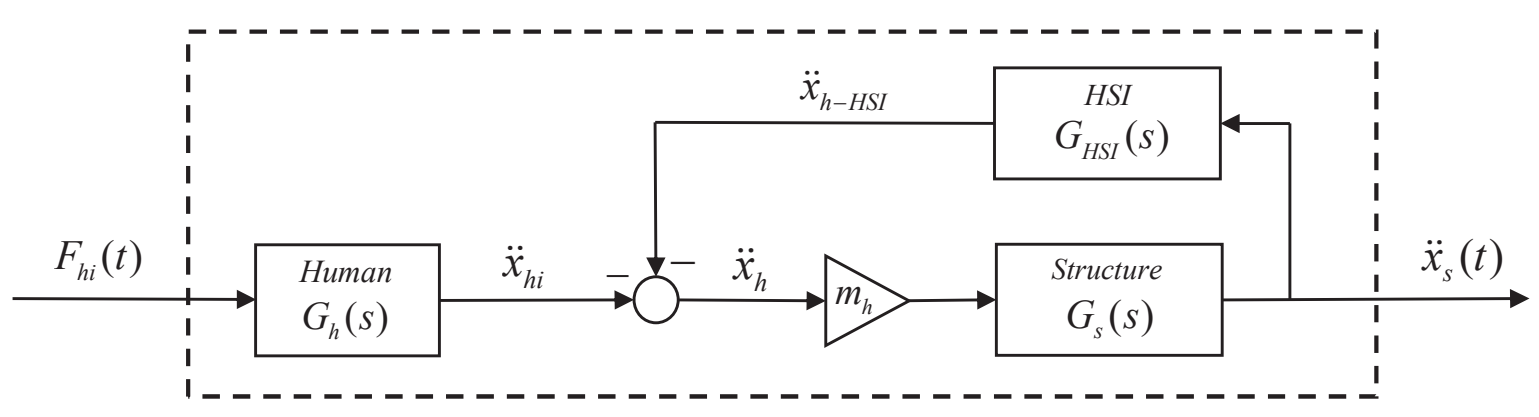

Figure 8: Closed-loop scheme for modelling HSI.

The main idea under this formulation is that the external force entering the structural system is equal to the inverse of the inertial force experienced by the human in real time. Thus, the human acceleration can be decomposed into two different components, one due to its motion $\ddot{x}_{h i}$, and a second human acceleration caused by the structural motion $\ddot{x}_{h-H S I}$. The first one can be directly obtained from the human actuator force by using the transfer function $G_{h}(s)$. The second is obtained using the transfer function $G_{H S I}(s)$ from the structural acceleration. By summing up the two components and multiplying them by the negative human mass, the external force on the structural system is derived, and so, the interaction loop is closed.

\subsection{Tuned Vibration Absorber}

The TVA can also be modelled as a closed feedback loop. The same idea used to model the HSI is now applied to the TVA. Hence, the external force entering the structural system due to the TVA is equal to the inverse of the inertial force of the TVA. The following transfer function similar to the one used for the HSI is defined as:

$$
G_{T V A}(s)=\frac{s^{2} X_{T}(s)}{s^{2} X_{s}(s)}=\frac{c_{T} s+k_{T}}{m_{T} s^{2}+c_{T} s+k_{T}}=\frac{2 \omega_{T} \zeta_{T} s+\omega_{T}^{2}}{s^{2}+2 \omega_{T} \zeta_{T} s+\omega_{T}^{2}}
$$

where $\omega_{T}=2 f \pi f_{T}$ is the circular natural frequency of the TVA $(\mathrm{rad} / \mathrm{s}), f_{T}$ is the respective natural frequency in $\mathrm{Hz}$ and $m_{T}, k_{T}$ and $\zeta_{T}$ are the mass $(\mathrm{kg})$, stiffness $(\mathrm{N} / \mathrm{m})$ and damping ratio of the TVA and $c_{T}=2 \omega_{T} \zeta_{T} m_{T}$. 
The dynamic system is now composed of a two-closed loops scheme, as shown in Figure 9.

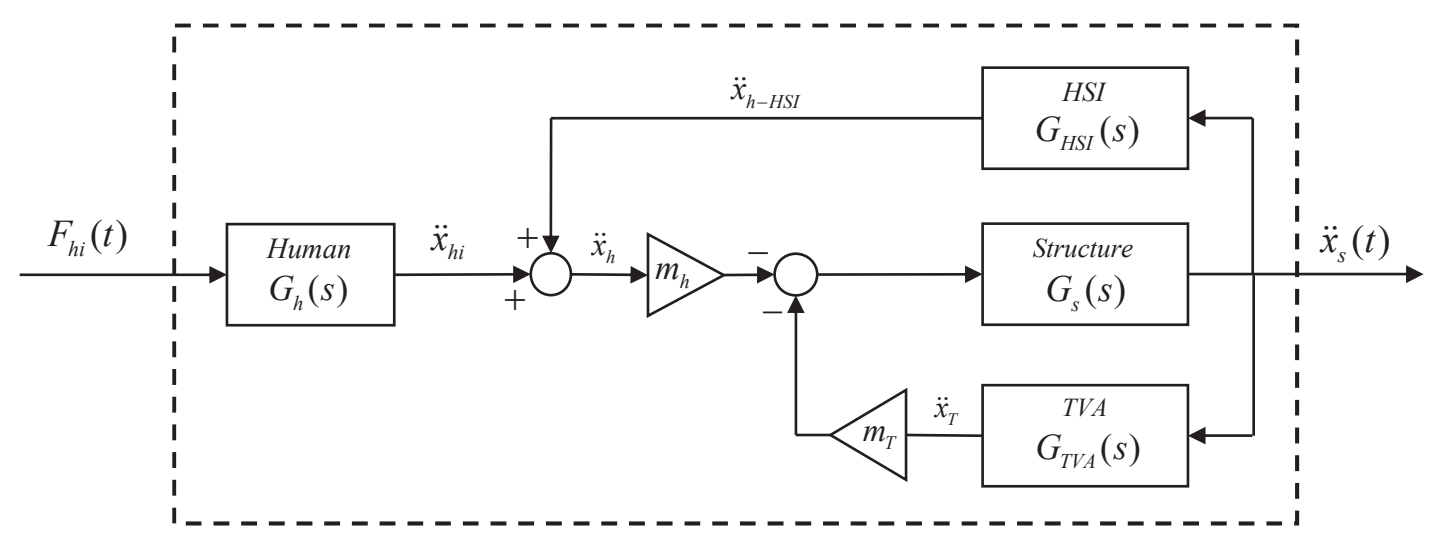

Figure 9: Double closed-loop scheme to model the structure with a TVA and HSI.

The TVA parameters have been designed using the approximation provided by Asami and Nishihara [12], based on the $H_{\infty}$ for primary structural systems with negligible damping. The following expressions provide the optimal $\omega_{T}$ and $\zeta_{T}$ which minimizes the structure acceleration for a given $m_{T}$ :

$$
\begin{aligned}
\eta & =\sqrt{\frac{1}{1+\mu}} \\
\zeta_{\mathrm{T}} & =\sqrt{\frac{3 \mu}{8(1+\mu)^{3}}} \cdot \sqrt{1+\frac{27}{32} \mu},
\end{aligned}
$$

where $\mu=m_{T} / m_{S}$ is the absorber-to-the-primary-system mass ratio. A mass ratio of $2 \%$ which corresponds to $m_{T}=13 \mathrm{~kg}$ has been selected. Parameter $\eta=\omega_{T} / \omega_{S}$ is the absorber-tothe-primary-system frequency ratio, in this case 0.99 . Finally, $\zeta_{T}$ is $8.7 \%$. The TVA has been designed following an optimum law which does not consider the influence of the HSI.

\subsection{Semi-Active Tuned Vibration Absorber}

The STVA has been designed using an on-off phase control-strategy [13]. This control strategy has been adopted due to its simplicity for implementing it in practical applications. Indeed, the real-time parameters required for this phase control are easy to measure: the structure acceleration, instead of the displacement, and the inertial mass velocity. The structural response is minimised when the velocity of the inertial mass $\dot{x}_{T}$ and the structural acceleration $\ddot{x}_{s}$ have opposite phases. Thus, the inertial controller objective is to facilitate a mass motion as close as possible to this phase. The aforementioned behaviour is equivalent to a $90^{\circ}$ phase lag between the structural acceleration and the control force. Thus, when the whole system behaves as desired, the viscous damping of the STVA should be small (ideally zero), however when the phase behaviour is not the correct one, the damper is blocked introducing a control force into the system [14]. The following phase control is summed up as follows:

$$
\left\{\begin{array}{lll}
\ddot{x}_{S} \cdot \dot{x}_{T} \leq 0 \quad \Rightarrow \quad c_{T}=c_{\min } & \text { (normal functioning) } \\
\ddot{x}_{S} \cdot \dot{x}_{T}>0 \quad \Rightarrow \quad c_{T}=c_{\max } & \text { (blocking functioning) },
\end{array}\right.
$$


where $c_{\min }$ is the minimum viscous damping given the minimum damper force when the STVA is working properly. The lower $c_{\min }$ results in better performance of STVA. Parameter $c_{\max }$ is the maximum viscous damping providing the maximum damper force which corresponds to the state of damper blocked. The magnitude $\ddot{x}_{S}$ needs to be measured using an accelerometer and $\dot{x}_{T}$ might be estimated from the integration of an accelerometer signal installed on the inertial mass. Both signals are low-pass filtered in order to avoid control instabilities related to the onoff control law. Note that the design parameters of the STVA correspond to those calculated for the TVA except for $c_{\min }$ and $c_{\max }$ that are equal to 0.1 and 50 times the value of the $c_{T}$ for the TVA, respectively. Figure 10 illustrates the non-linear closed loop used to model the STVA, in presence of HSI. Note that if $c_{T}$ is constant, then block diagrams of Figures 9 and 10 become the same.

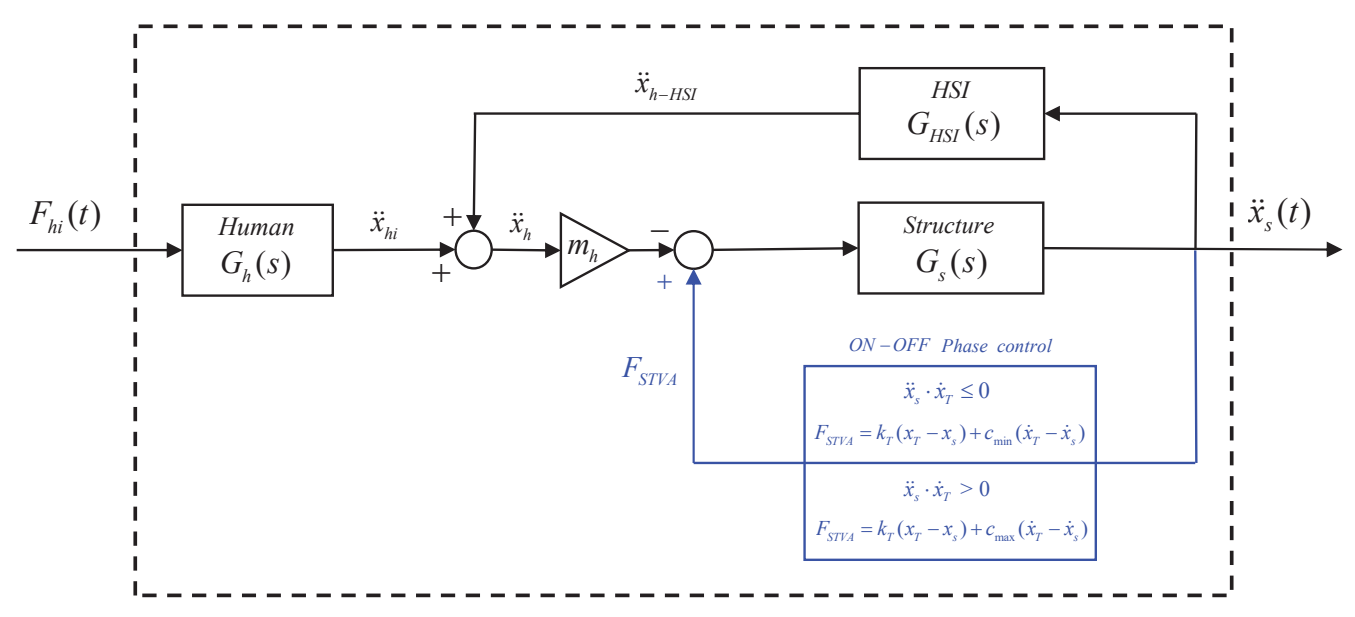

Figure 10: Double closed-loop scheme to model the structure with a STVA considering the HSI.

\subsection{Active Vibration Absorber}

Direct Velocity Feedback (DVF) control strategy has been used for the AVA. This means that the control force introduced into the system using an actuator is in phase with structure's velocity. Although DVF is, by its nature, unconditionally stable, when accounting for the actuator dynamics, the closed-loop system becomes conditionally stable and the stability margin has to be studied prior to the implementation [15]. The velocity estimated is multiplied by a control gain $K_{C}$ producing a command signal in terms of voltage to the actuator. Firstly, the limit control gain for stability is derived by classical root locus techniques, $K_{C_{\text {lim }}} \simeq 70$. Finally, a control gain $K_{C}$ equal to $K_{C_{\text {lim }}} / 2$ has been chosen providing enough stability gain margin and leading to a safe implementation [16]. The actuator transfer function between the transmitted control force and the control voltage is considered as a second-order proof-mass actuator model:

$$
G_{A V A}(s)=\frac{150 s^{2}}{s^{2}+5.7 s+8.2^{2}}
$$

where the circular natural frequency of the actuator is $\omega_{A}=8.2 \mathrm{rad} / \mathrm{s}(1.3 \mathrm{~Hz})$ and $2 \zeta_{a} \omega_{a}=5.7$, $\zeta_{a}$ being the actuator damping ratio. The chosen AVA has an inertial mass equal of $13 \mathrm{~kg}$, the same as the one used for the TVA and STVA. The natural frequency of the actuator is a critical parameter when controlling low-frequency vibrations. The lower the natural frequency of the 
actuator is, the closer to linear the behaviour of the actuator is. A sufficiently small, but realistic, value for the actuator passive behaviour in terms of natural frequency has been chosen. Note that the shaker's natural frequency can be changed by means of changing the passive stiffness or by the use of more sophisticated control laws [17]. Figure 11 depicts the feedback scheme for the AVA.

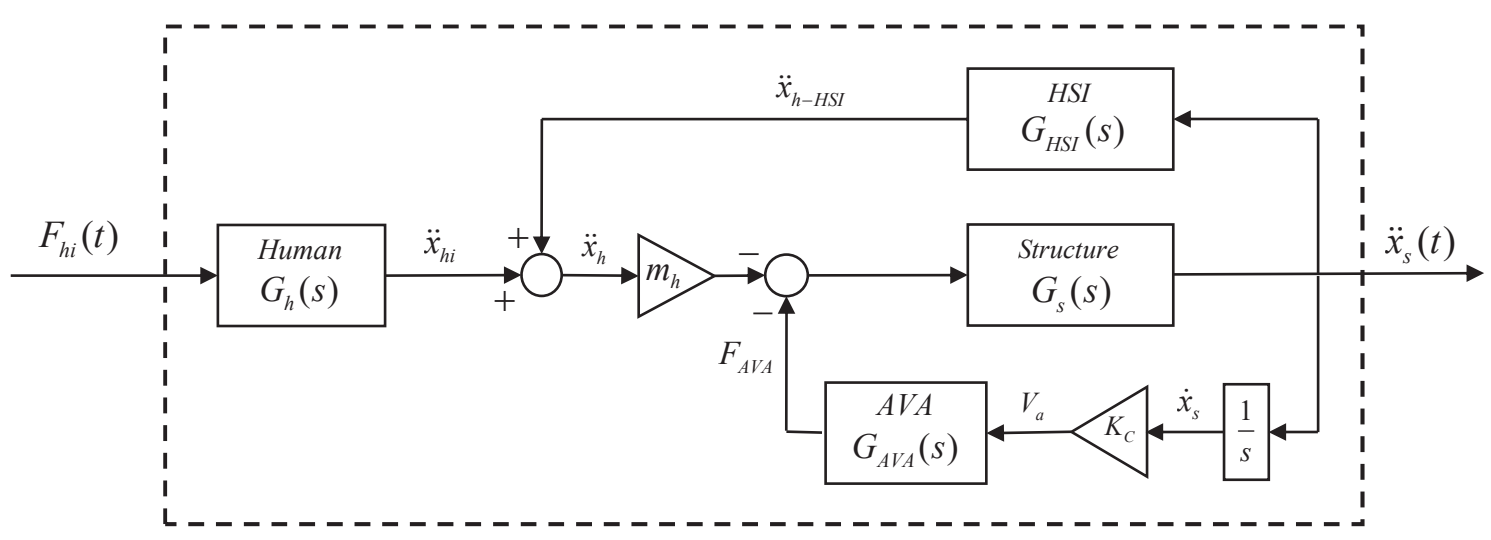

Figure 11: Double closed-loop scheme to model the structure with a AVA considering the HSI

\section{RESULTS}

Figures 12 and 13 provide the resonant response of the structural system under bouncing action without and with HSI, respectively. Three serviceability indicators are calculated: the Peak acceleration value, the maximum value of the running $1 \mathrm{~s}$ root mean square acceleration (often refered to as Maximum Transient Vibration Value or MTVV) and the cumulative Vibration Dose Value (VDV).

When assessing the structural response at resonance, the accelerations reached without considering HSI are not only excessive but also unlikely to be achieved. Once the HSI is taken into account, the dynamic response decreases significantly. In both cases, the structure exceeds the vibration serviceability limit of $1 \mathrm{~m} / \mathrm{s}^{2}$ for peak acceleration. Therefore there is a need to apply inertial vibration controllers to reduce the structural vibration.

When including HSI into the dynamic analysis, the system to be controlled is no longer the structure. The human is a new element to be considered as is influences the dynamic properties of the system to be controlled. The inertial vibration controllers reduce their damping capacity at resonance (especially the TVA and STVA), due to part of the energy introduced into the structure is damped by the very human system which generates it. This fact can be observed in Figure 14 where the VDV of all the dynamic loading cases are compared. The VDV is a suitable vibration serviceability parameter to use for this comparison because of its cumulative nature. To explain the controllers performance out of resonance is a bit more complicated and would require an overall and deep comprehension of the interaction phenomena between all the elements that compound the dynamic system.

The AVA is by far the most effective of the three inertial controllers considered, and it is the only solution capable of reducing the peak acceleration at resonant bouncing below the established threshold of $1 \mathrm{~m} / \mathrm{s}^{2}$ (Figure 13d). Therefore, active control is the most promising damping technique for controlling ultra-weight structures, as it is able to effectively cancel complex dynamic responses compound of resonant and non-resonant harmonics. 


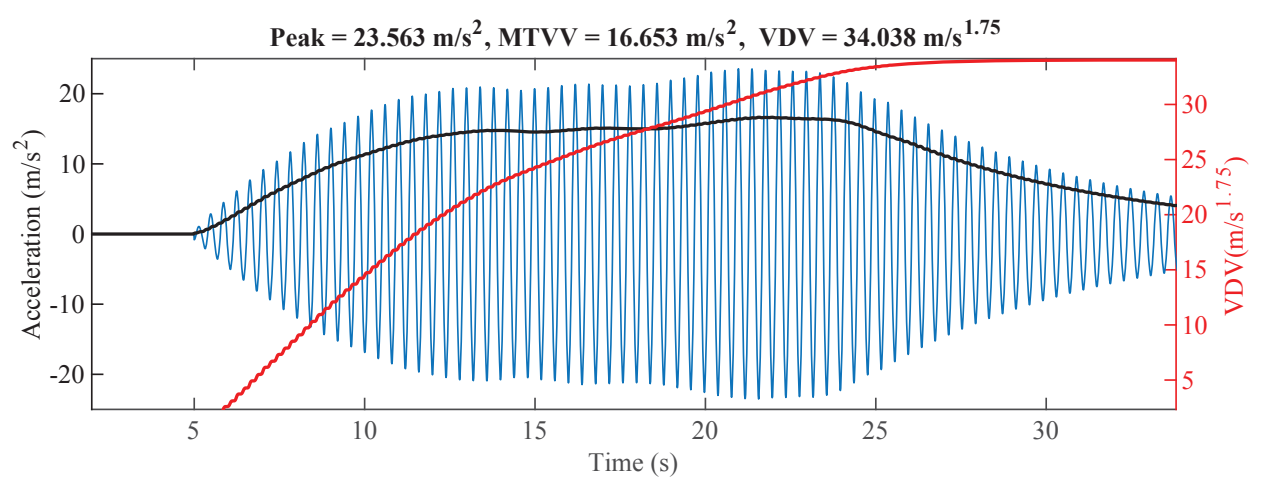

(a) Uncontrolled.

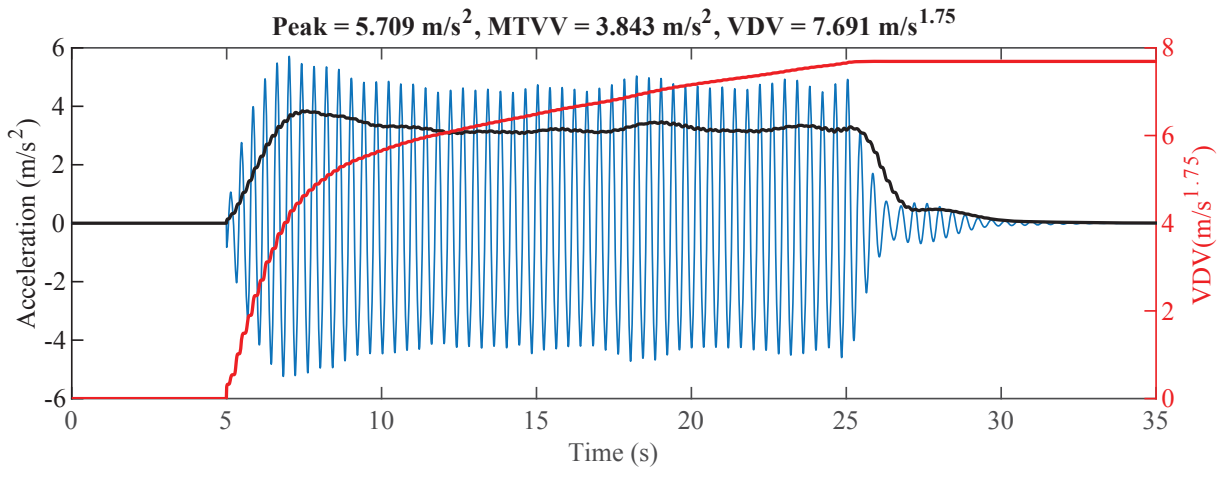

(b) Passive.

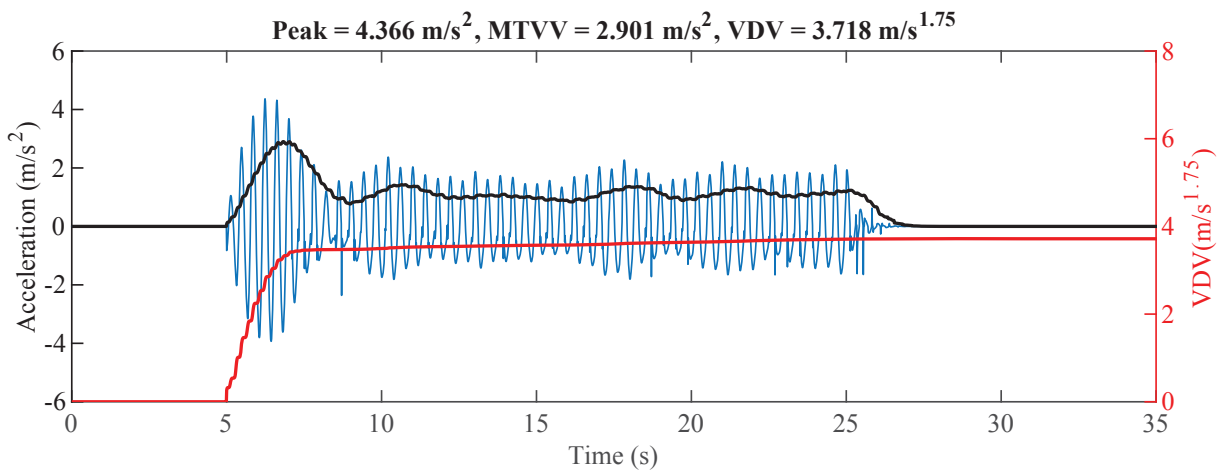

(c) Semi-active.

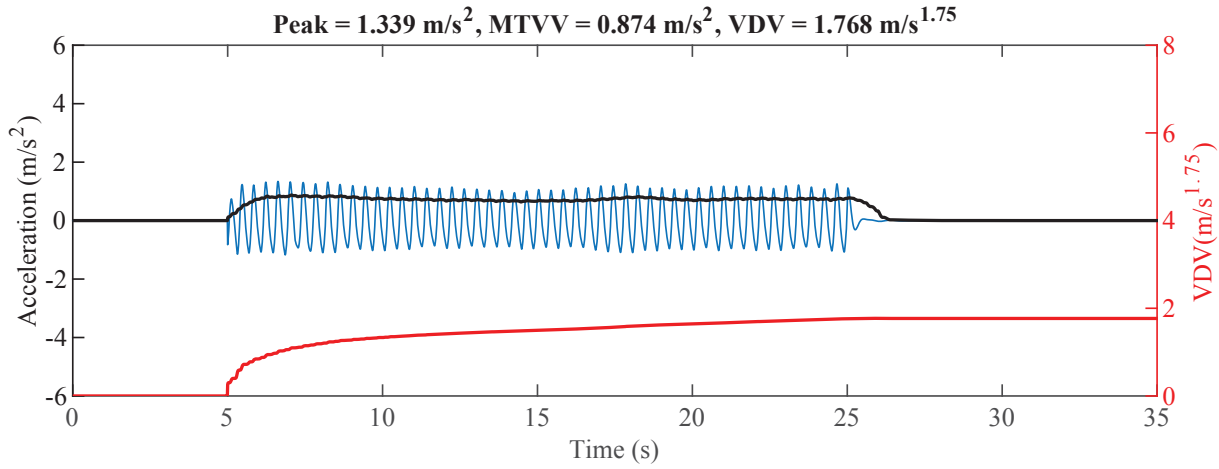

(d) Active.

Figure 12: Resonance response of the structure without HSI under bouncing loading. 


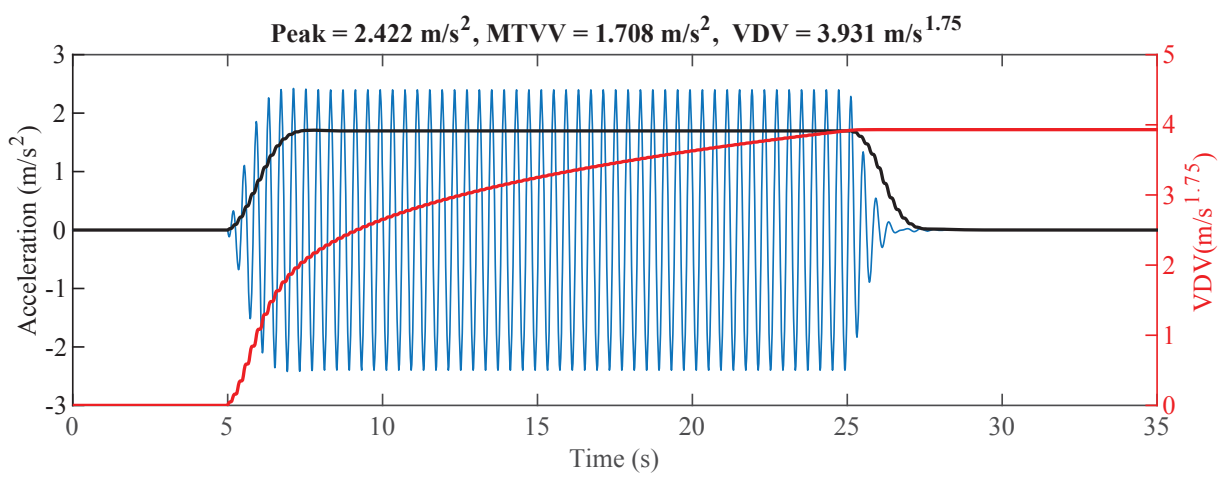

(a) Uncontrolled.

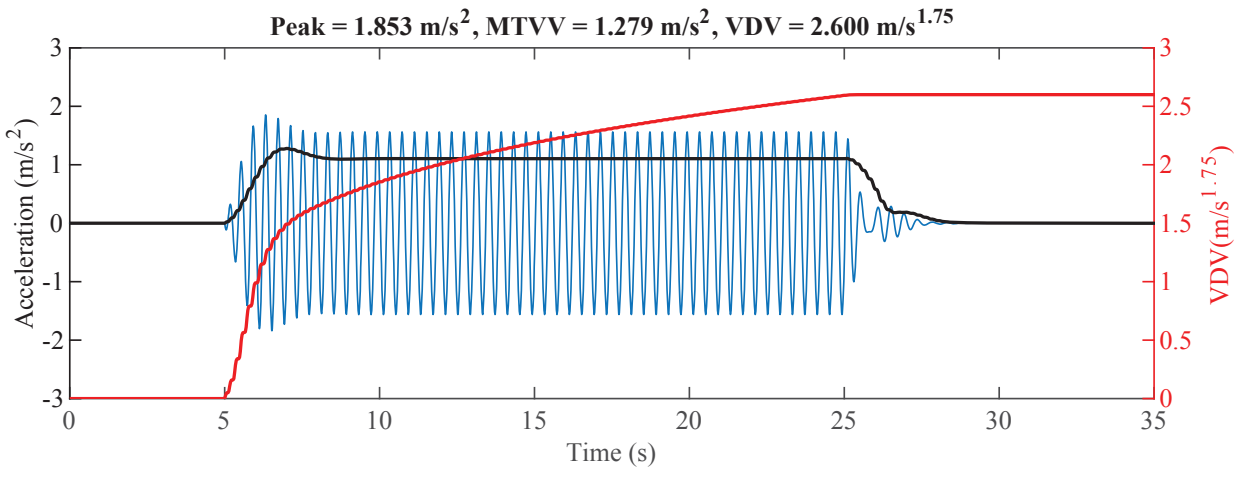

(b) Passive.

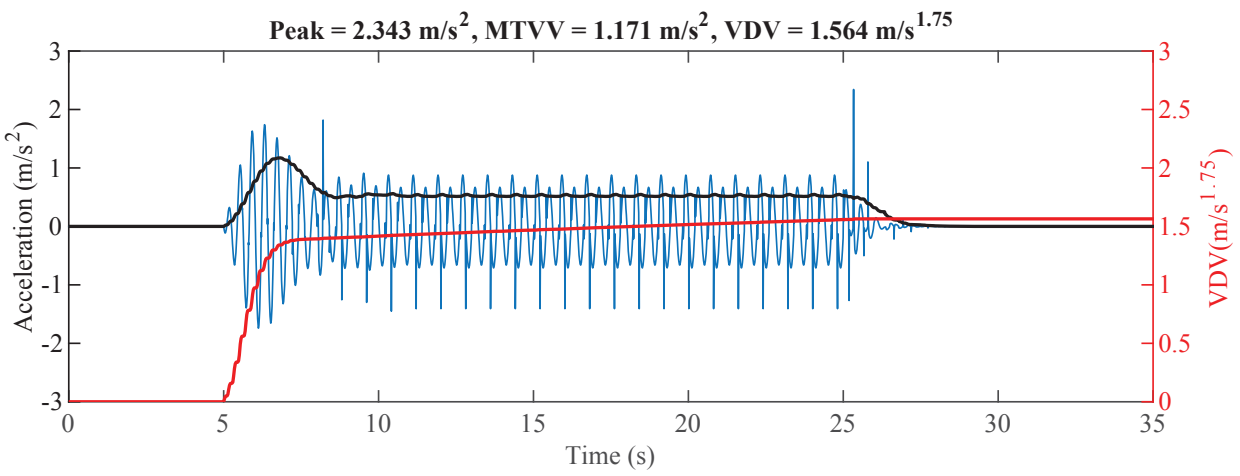

(c) Semi-active.

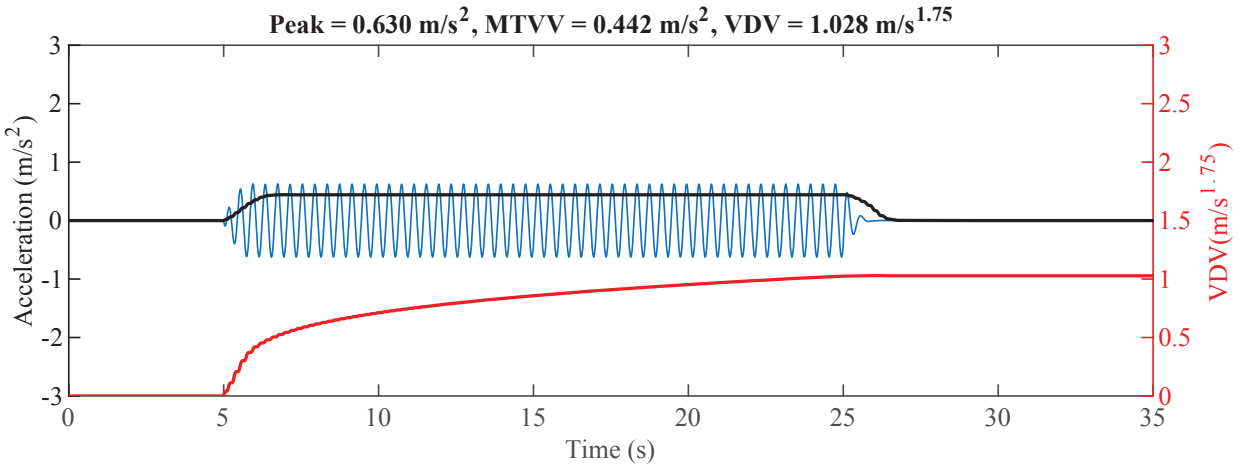

(d) Active.

Figure 13: Resonance response of the structure with HSI under bouncing loading. 


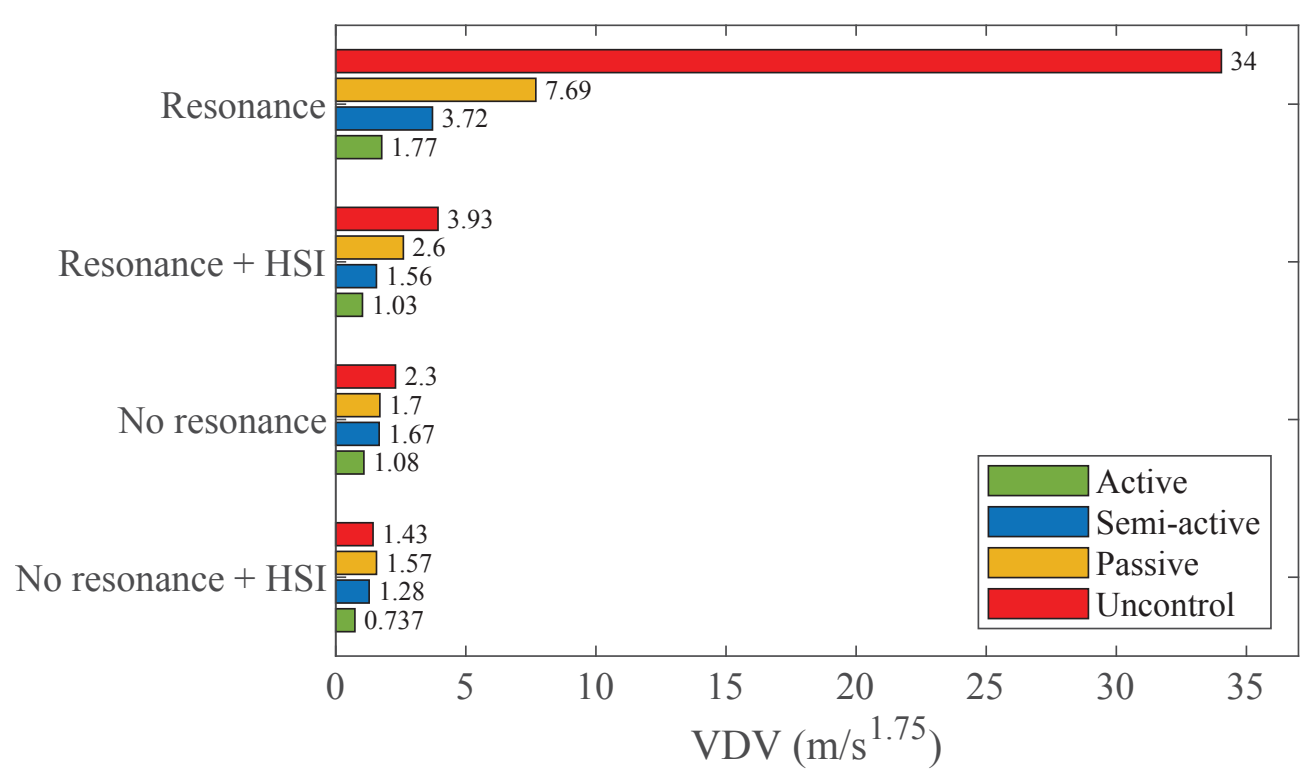

Figure 14: VDV of the structural response for all loading cases.

\section{CONCLUSIONS}

In this paper, three types of inertial controllers (Passive, Semi-active and Active) have been applied to an ultra-lightweight FRP footbridge conceived as a unique laboratory facility to investigate the HSI. The dynamic response of the first flexural vibration mode of the structure and the controllers' performance have been numerically studied in the presence and absence of HSI.

The uncontrolled case without HSI leads to an unrealistic acceleration of $23.5 \mathrm{~m} / \mathrm{s}^{2}$. However, when the HSI is included the acceleration is reduced to a realistic value of $2.4 \mathrm{~m} / \mathrm{s}^{2}$. Moreover, when HSI is included, the controllers reduce drastically their performance, mainly due to the fact that they were designed without taking into account the HSI. The improvements for the resonant case (with interaction) were $34 \%, 60 \%$ and $74 \%$ for passive, semi-active and active, respectively in terms of VDV. However for non-resonant excitation, the only controller able to mitigate vibration was the AVA with a $50 \%$ of improvement.

This paper is a first study on the performance of inertial controllers for human-induced excitation of structures prone to show HSI. Future works on the design of inertial controllers should include the HSI and the resulting human-structure model should be carefully analyzed in order to propose new controller design strategies for these unexplored systems.

\section{ACKNOWLEDGEMENTS}

The authors acknowledge the financial support provided by the UK's Engineereing and Physical Sciences Reasecrh Council (project EP/M021505/1: Characterising dynamic performance of fibre reinforced polymer structures for resilience and sustainability) and the Spanish Ministry of Science, Innovation and Universities through the project SEED-SD (RTI2018-099639B-I00). Dr Iván M. Díaz would like to acknowledge the financial support provided by Fundación José Entrecanales Ibarra to carry out a Research Stay at University of Exeter. Carlos M. C. Renedo would like to thank Universidad Politécnica de Madrid for the financial support through a $\mathrm{PhD}$ research grant. 


\section{REFERENCES}

[1] D. Kendall, Next Generation of footbridges in FRP composites, Proceedings of Footbridge 2014 - Past, present and future, 16-18 July, London, United Kingdom, 1-11, 2014.

[2] C. M. C. Renedo, I. M. Díaz and J. H. García-Palacios, High performance dynamicallyloaded structures: integrating smart dampers, Proceedings of International fib Symposium on Conceptual Design of Structures, 26-28 September, Madrid, Spain, 33-40, 2019.

[3] J. Russell, J. T. Mottram, S. Živanović and X. Wei, Design and Performance of a lively FRP footbridge, Volume 2: Proceedings of the 37th IMAC, A Conference and Exposition on Structural Dynamics, 28-31, January, Orlando, Florida, 125-128, 2019.

[4] J. F. Jímenez-Alonso, A. C. Sáez, E. Caetano and F. Magalhaes, Vertical crowd-structure interaction model to analyze the change of the modal properties of a footbridge, Journal of bridge Engineering, 21(8), 1-19, 2016.

[5] D. Nyawako and P. Reynolds, Technologies for mitigation of human-induced vibrations in civil engineering structures. The Shock and Vibration Digest, 39 (6),465-493, 2007.

[6] H. Bachmann and B. Weber, Tuned Vibration Absorbers for Damping of "Lively Structures", Structural Engineering International, 95 (1), 31-37, 1995.

[7] M. Setareh, Floor vibration control using semi-active tuned mass dampers, Canadian Journal of Civil Engineering 29, 76-84, 2002.

[8] L. Hanagan and T. Murray, Active control approach for reducing floor vibrations, Journal of Structural Engineer 123 (11), 1497-1505,1997.

[9] X. Wei, J. Russell, S. Zivanovic and J.T. Mottram, Measured dynamic properties for FRP footbridges and their critical comparison against structures made of conventional construction materials, Composite Structures, 223, 1-16, 2019.

[10] S. Živanović, J. Russell and V. Racic, Vibration performance of a lightweight FRP footbridge under dynamic excitation by pedestrians, Volume 2: Proceedings of the 37th IMAC, A Conference and Exposition on Structural Dynamics, 28-31, January, Orlando, Florida, 111-114, 2019.

[11] J. W. Dougill, J. R. Wright, J. G. Parkhouse and R. E. Harrison, Human structure interaction during rhythmic bobbing, The Structural Engineer, 84 (22), 32-39, 2006.

[12] T. Asami, and O. Nishihara, Closed-form exact vibration absorbers (Application to different transfer functions) and damping systems, Journal of Vibration and AcousticsTransactions of the Asme, 125 (3), 398-405, 2003.

[13] C. Moutinho, Testing a simple control law to reduce broadband frequency harmonic vibrations using semi-active tuned mass dampers, Smart Materials and Structures, 24 (5), 05507, 2015.

[14] J. M. Soria, I. M. Díaz and J. H. García-Palacios, Further steps towards the tuning of inertial controllers for broadband-frequency-varying structures, Structural Control and Health Monitoring, 27, 10.1002/stc.2461, 2020. 
[15] I. M. Díaz and P. Reynolds, Robust saturated control of human-induced vibrations via a proof-mass actuator, Smart Material and Structures, 18 (12), 125024-125033, 2009.

[16] X. Wang, E. Pereira, J. H. García-Palacios and I. M. Díaz, A general vibration control methodology for human induced vibrations, Structural Control and Health Monitoring, 26 (10), 10.1002/stc.2406, 2019.

[17] I. M. Díaz, E. Pereira, M. J. Hudson and P. Reynolds, Enhancing active vibration control of pedestrian structures using inertial actuators with local feedback control, Engineering Structures, 41, 157-166, 2012. 